\title{
Stability and periodicity in a mosquito population suppression model composed of two sub-models
}

\author{
Zhongcai Zhu • Bo Zheng · Yantao Shi • \\ Rong Yan · Jianshe Yu
}

Received: 30 June 2021 / Accepted: 9 November 2021 / Published online: 1 December 2021

(C) The Author(s) 2021

\begin{abstract}
In this paper, we propose a mosquito population suppression model which is composed of two sub-models switching each other. We assume that the releases of sterile mosquitoes are periodic and impulsive, only sexually active sterile mosquitoes play a role in the mosquito population suppression process, and the survival probability is density-dependent. For the release waiting period $T$ and the release amount $c$, we find three thresholds denoted by $T^{*}, g^{*}$, and $c^{*}$ with $c^{*}>g^{*}$. We show that the origin is a globally or locally asymptotically stable equilibrium when $c \geq c^{*}$ and $T \leq T^{*}$, or $c \in\left(g^{*}, c^{*}\right)$ and $T<T^{*}$. We prove that the model generates a unique globally asymptotically stable $T$-periodic solution when either $c \in\left(g^{*}, c^{*}\right)$ and $T=T^{*}$, or $c>g^{*}$ and $T>T^{*}$. Two numerical examples are provided to illustrate our theoretical results.
\end{abstract}

Keywords Sterile mosquitoes - Mosquito population suppression - Periodic solutions · Asymptotic stability · Impulsive and periodic release

Mathematics Subject Classification 34C25 -34D20 . 34D23 - 92D25 - 93D20

Z. Zhu · B. Zheng · Y. Shi · R. Yan · J. Yu (凶)

Guangzhou Center for Applied Mathematics, Guangzhou University, Guangzhou 510006, China

e-mail: jsyu@gzhu.edu.cn

\section{Introduction}

To prevent and control the spread of mosquito-borne diseases such as malaria, dengue fever and Zika, effective and biologically safe approaches are to suppress the wild mosquito populations by using the sterile insect technique (SIT), the genetic approaches, and the Wolbachia-driven mosquito control technique, in which sterile mosquitoes, including irradiated and Wolbachia-infected male mosquitoes, are released into the wild to mate with wild female mosquitoes [1-6]. Various mathematical models have been developed to study the dynamics of mosquito populations with the release of sterile mosquitoes, we refer to [7-24] and references therein.

A wild female mosquito mating with a sterile mosquito will not be capable of producing offspring. Since the only function of the released males is to sterilize wild mosquitoes, only those sexually active sterile mosquitoes may play a role in the suppression process of wild mosquitoes. Based on these observations, the author in [17] first gave a novel modeling idea by treating the number of sterile mosquitoes as a given function instead of an independent variable satisfying a dynamical equation to explore the interactive dynamics of wild and sterile mosquitoes. This modeling technique mathematically reduces a planar system to a scalar differential equation, which makes mathematical analysis more tractable $[18,19,25,26]$.

Due to the facts that mosquitoes have four metamorphic stages of development during their lifetime and the 
intraspecific competition mainly occurs in the aquatic stages and thus in the birth process [27,28], the author in [29] proposed the following model:

$$
\begin{aligned}
& \frac{d w}{d t}=\frac{a w}{w+g}(1-\xi w) w-\mu w, \\
& \frac{d g}{d t}=\mathbb{B}(\cdot)-\mu g,
\end{aligned}
$$

where $w=w(t)$ and $g=g(t)$ are the numbers of wild and sterile mosquitoes at time $t$, respectively, $a$ is the maximum number of survived offspring produced per mosquito, $\frac{w}{w+g}$ is the mating probability between wild mosquitoes, $\mu$ is the density-independent death rate of wild mosquitoes, $\xi$ is the carrying capacity parameter such that $1-\xi w$ describes the density-dependent survival probability, and $\mathbb{B}(\cdot)$ is the release rate of the sterile mosquitoes.

Applying the modeling idea in [17], the authors in [30] discarded the second equation of system (1.1) by treating $g(t)$ as a given nonnegative continuous function in advance, and formulated the following nonautonomous ordinary differential equation model:

$$
\frac{d w}{d t}=\frac{a w}{w+g}(1-\xi w) w-\mu w .
$$

The strategy of periodic and impulsive releases of sterile mosquitoes has been proposed and well studied in $[18,19,25,26,31]$, where only sexually active mosquitoes are included in the models. Let $\bar{T}$ and $T$ be the sexual lifespan of sterile mosquitoes and the waiting period between two consecutive releases, respectively. Accordingly, there are three possible release strategies: $T<\bar{T}, T=\bar{T}$, and $T>\bar{T}$. For the release strategy of $T=\bar{T}, g(t) \equiv c$, model (1.2) becomes

$$
\frac{d w}{d t}=\frac{a w}{w+c}(1-\xi w) w-\mu w .
$$

With the introduction of the release amount threshold

$g^{*}=\frac{(a-\mu)^{2}}{4 a \mu \xi}$,

the authors in [30] gave the following theorem.

Theorem 1.1 For model (1.3), the following statements are true.

(1) For the case when $c \in\left(0, g^{*}\right)$, model (1.3) has three equilibria: the origin, denoted by $w_{0}$, and two positive equilibria

$$
\begin{aligned}
& w_{1}(c)=\frac{A}{2}-\sqrt{\frac{\mu}{a \xi}\left(g^{*}-c\right)} \text { and } \\
& w_{2}(c)=\frac{A}{2}+\sqrt{\frac{\mu}{a \xi}\left(g^{*}-c\right),}
\end{aligned}
$$

where $A=\frac{a-\mu}{a \xi}$. Furthermore, $w_{0}$ and $w_{2}(c)$ are both asymptotically stable, and $w_{1}(c)$ is unstable.

(2) For the case when $c=g^{*}$, model (1.3) admits $w_{0}$ and $w^{*}=\frac{A}{2}$ as the equilibria. $w_{0}$ is asymptotically stable, but $w^{*}$ is semi-stable, stable from the rightside and unstable from the left-side.

(3) For the case when $c>g^{*}$, model (1.3) admits a unique equilibrium $w_{0}$, which is globally asymptotically stable.

In this paper, we focus on the release strategy of $T>\bar{T}$, which means that the sterile mosquitoes previously released have lost their mating competitiveness before the release of the next batch of sterile mosquitoes. We assume that a constant amount $c$ of sterile mosquitoes are released at discrete time points $T_{i}=i T, i=0,1,2, \ldots$ During each $T$-periodic interval $[i T,(i+1) T)$, the released mosquitoes at time $T_{i}$ are sexually active for $t \in[i T, i T+\bar{T})$, but inactive for $t \in[i T+\bar{T},(i+1) T), i=0,1,2, \ldots$ We find that $g$ follows the following $T$-periodic and piecewise constant function:

$g=\left\{\begin{array}{ll}c, & \text { for } t \in[i T, i T+\bar{T}), \\ 0, & \text { for } t \in[i T+\bar{T},(i+1) T),\end{array} \quad i=0,1,2, \ldots\right.$

Then, model (1.2) is divided into the following two sub-equations:

$$
\begin{gathered}
\frac{d w}{d t}=\frac{a w^{2}}{w+c}(1-\xi w)-\mu w, \\
t \in[i T, i T+\bar{T}),
\end{gathered}
$$

and

$$
\begin{aligned}
\frac{d w}{d t}= & -a \xi w(w-A), \\
& t \in[i T+\bar{T},(i+1) T),
\end{aligned}
$$

which constantly switch each other, where $i=$ $0,1,2, \ldots$. We assume $a>\mu$ throughout this paper, so that the wild mosquito population without the interference of sterile mosquitoes is stabilized at the population size $A[25,32,33]$. 
Define

$T^{*}=\frac{a}{a-\mu} \bar{T}$ and $\quad c^{*}=\frac{a-\mu}{\mu \xi}$

as the release period threshold and the second release amount threshold, respectively. Obviously, we have $c^{*}>g^{*}$. In this paper, we investigate the existence, uniqueness and stability of $T$-periodic solutions to model (1.4)-(1.5) by using Poincaré map method. These results may be helpful for optimal control of mosquito vector or mosquito-borne diseases as shown in [34]. In Sect. 2, we derive the Poincaré map formulation by solving (1.4)-(1.5) with initial value. We prove in Sect. 3 that the origin is globally asymptotically stable for the case when $c \geq c^{*}$ and $T \leq T^{*}$. The origin is locally asymptotically stable if and only if $g^{*}<c<c^{*}$ and $T<T^{*}$, and model (1.4)-(1.5) has a unique globally asymptotically stable $T$-periodic solution for the case when $g^{*}<c<c^{*}$ and $T=T^{*}$, or $c>g^{*}$ and $T>T^{*}$. Two numerical examples are also given to illustrate our theoretical results. Finally, in Sect. 4, we provide some discussions about the current and future work.

\section{Preliminaries}

It is clear to see that model (1.4)-(1.5) admits $w_{0}$ as the unique equilibrium. For any initial condition $\left(t_{0}, u_{0}\right) \in[0, \infty) \times(0, \infty)$, let $w(t)=w\left(t ; t_{0}, u_{0}\right)$ for $t \geq t_{0}$ denote the solution of model (1.4)-(1.5) with initial value $w\left(t_{0}\right)=u_{0}$. Then, $w\left(t ; t_{0}, u_{0}\right)$ is a continuous and piecewise differentiable function defined on $\left[t_{0}, \infty\right)$. When $t_{0}=0$, solution $w(t ; 0, u)$ follows Eq. (1.4) satisfying $w(0)=u$ on $[0, \bar{T})$, and then initiated from $w(\bar{T})=w\left(\bar{T}^{-}\right)$, the solution goes to $w(T ; 0, u)$ following Eq. (1.5) for $t \in[\bar{T}, T)$. The solution can be described on the other $T$-periodic intervals in the same manner. According to the continuous and differentiable dependence of the solutions on the initial values to ordinary differential equations [35], we obtain that $w(\bar{T} ; 0, u)$ and $w(T ; 0, u)$ are both continuously differentiable with respect to $u$. Furthermore, if $w(T)=w(T ; 0, u)=u$, then $w(t)=w(t ; 0, u)$ is a $T$-periodic solution of model (1.4)-(1.5). Thus, in order to find a $T$-periodic solution of model (1.4)-(1.5), we only need to find an initial value $u>0$ such that $w(T ; 0, u)=u$. We write

$h(u)=w(T ; 0, u), \quad \bar{h}(u)=w(\bar{T} ; 0, u)$.
Then, $h(u)=w(T ; \bar{T}, \bar{h}(u))$.

To obtain $\bar{h}(u)$ and $h(u)$, we need to solve initial value problem (1.4) with $w(0)=u$. Now, we assume $c>g^{*}$. Equation (1.4) can be rewritten as:

$\frac{d w}{d t}=\frac{-a \xi w}{w+c}\left(w^{2}-A w+\frac{\mu c}{a \xi}\right)$,

or, equivalently,

$-a \xi d t=\left(\frac{\alpha}{w}+\frac{\beta\left(w-\frac{A}{2}\right)+\gamma}{\left(w-\frac{A}{2}\right)^{2}+b^{2}}\right) d w$,

which yields

$d\left\{\ln \left[w^{\alpha}\left(\left(w-\frac{A}{2}\right)^{2}+b^{2}\right)^{\frac{\beta}{2}} \exp \left(\frac{\gamma}{b} \tan ^{-1}\left(\frac{w-\frac{A}{2}}{b}\right)\right)\right]\right\}$
$=-a \xi d t$,

where

$\alpha=\frac{a \xi}{\mu}, \quad \beta=-\frac{a \xi}{\mu}, \gamma=\frac{a+\mu}{2 \mu}, b=\sqrt{\frac{\mu}{a \xi}\left(c-g^{*}\right)}$.

Integrating (2.2) from 0 to $\bar{T}$, we obtain

$$
\begin{gathered}
w^{\alpha}(\bar{T})\left(\left(w(\bar{T})-\frac{A}{2}\right)^{2}+b^{2}\right)^{\frac{\beta}{2}} \\
\exp \left(\frac{\gamma}{b} \tan ^{-1}\left(\frac{w(\bar{T})-\frac{A}{2}}{b}\right)\right) \\
=w^{\alpha}(0)\left(\left(w(0)-\frac{A}{2}\right)^{2}+b^{2}\right)^{\frac{\beta}{2}} \\
\exp \left(\frac{\gamma}{b} \tan ^{-1}\left(\frac{w(0)-\frac{A}{2}}{b}\right)\right) e^{-a \xi \bar{T}},
\end{gathered}
$$

which yields, from (2.1),

$$
\begin{gathered}
\bar{h}^{\alpha}(u)\left(\left(\bar{h}(u)-\frac{A}{2}\right)^{2}+b^{2}\right)^{\frac{\beta}{2}} \\
\exp \left(\frac{\gamma}{b} \tan ^{-1}\left(\frac{\bar{h}(u)-\frac{A}{2}}{b}\right)\right) \\
=u^{\alpha}\left(\left(u-\frac{A}{2}\right)^{2}+b^{2}\right)^{\frac{\beta}{2}} \\
\exp \left(\frac{\gamma}{b} \tan ^{-1}\left(\frac{u-\frac{A}{2}}{b}\right)\right) e^{-a \xi \bar{T}} .
\end{gathered}
$$


Now, we solve initial value problem (1.5) for $t \in$ $[\bar{T}, T)$ with $w(\bar{T})=w\left(\bar{T}^{-}\right)=\bar{h}(u)$. It follows from (1.5) that

$d\left(\ln \left(\frac{w}{|A-w|}\right)\right)=a A \xi d t$.

Integrating (2.4) from $\bar{T}$ to $T$ yields

$\frac{w(T)}{A-w(T)}=\frac{w(\bar{T})}{A-w(\bar{T})} e^{a A \xi(T-\bar{T})}$,

and thus, we get, from (2.1),

$\frac{\bar{h}(u)}{A-\bar{h}(u)}=\frac{m h(u)}{A-h(u)}$,

where $m=e^{-a A \xi(T-\bar{T})}$.

Define function sequences $\left\{h_{n}\right\}$ and $\left\{\bar{h}_{n}\right\}$ as follows:

$h_{n}(u)=w(n T ; 0, u)$,

$\bar{h}_{n}(u)=w(n T+\bar{T} ; 0, u), \quad n=0,1,2, \ldots$

We obtain, by induction,

$\begin{aligned} h_{n+1}(u) & =w\left(T ; 0, h_{n}(u)\right)=h\left(h_{n}(u)\right), \\ \bar{h}_{n}(u) & =\bar{h}\left(h_{n}(u)\right), \quad n=0,1,2, \ldots,\end{aligned}$

where

$h_{0}(u)=u, \quad \bar{h}_{0}(u)=w(\bar{T} ; 0, u), \quad$ and

$h_{1}(u)=h(u)$.

By using a similar argument to that in [25], we can easily prove the next lemma.

Lemma 2.1 For any given initial value $u$, the following conclusions hold.

(1) Sequences $\left\{h_{n}(u)\right\}$ and $\left\{\bar{h}_{n}(u)\right\}$ are both strictly increasing if and only if $h(u)>u$.

(2) $h_{n}(u) \equiv u, n=1,2,3, \ldots$, if and only if $h(u)=u$. Hence, $w(t)=w(t ; 0, u)$ is a T-periodic solution of model (1.4)-(1.5).

(3) Sequences $\left\{h_{n}(u)\right\}$ and $\left\{\bar{h}_{n}(u)\right\}$ are both strictly decreasing if and only if $h(u)<u$.

Note that $w(t)=A$ is a solution of the second equation of model (1.4)-(1.5), along with $c>g^{*}$, we have $h(u)<u$, for $u \geq A$.

Thus, if there exists $\delta \in(0, A)$ such that $h(\delta)>\delta$, then model (1.4)-(1.5) has at least one $T$-periodic solution $w(t ; 0, u)$ with $u \in[\delta, A)$ owing to the continuous differentiability of $h(u)$.
Since $\bar{h}(u) \rightarrow 0$ as $u \rightarrow 0$, we obtain, from (2.3),

$\lim _{u \rightarrow 0} \frac{\bar{h}(u)}{u}=e^{-\frac{a \xi \bar{T}}{\alpha}}=e^{-\mu \bar{T}}$.

Following from $h(u) \rightarrow 0$ as $u \rightarrow 0$, we have, from (2.5),

$$
\begin{aligned}
\lim _{u \rightarrow 0} \frac{h(u)}{\bar{h}(u)} & =\lim _{u \rightarrow 0} \frac{A-h(u)}{m(A-\bar{h}(u))} \\
& =\frac{1}{m}=e^{a A \xi(T-\bar{T})}=e^{(a-\mu)(T-\bar{T})} .
\end{aligned}
$$

Therefore, from (2.7) and (2.8), we have

$$
\begin{aligned}
h^{\prime}(0) & =\lim _{u \rightarrow 0} \frac{h(u)}{u}=\lim _{u \rightarrow 0}\left(\frac{h(u)}{\bar{h}(u)} \cdot \frac{\bar{h}(u)}{u}\right) \\
& =e^{(a-\mu)\left(T-\frac{a}{a-\mu} \bar{T}\right)}=e^{(a-\mu)\left(T-T^{*}\right)} .
\end{aligned}
$$

Hence, $h^{\prime}(0)>1$ implies that model (1.4)-(1.5) has at least one $T$-periodic solution.

\section{Main results}

In this section, we will present our main results by dividing the study into two cases: $T \leq T^{*}$ and $T>T^{*}$.

\subsection{Release more often with $T \leq T^{*}$}

In this subsection, we consider the case of $T \leq T^{*}$. That is, we release sterile mosquitoes more often. Before presenting our first result, we give the following lemma.

Lemma 3.1 Assume that $c \geq c^{*}$ and $T \leq T^{*}$. Then, we have

$h(u)<u$, for $u>0$.

Proof By (2.6), to prove (3.1), we only need to prove

$h(u)<u$, for $u \in(0, A)$.

We divide the proof of (3.2) into two cases: $T=T^{*}$ and $T<T^{*}$. For the case when $T=T^{*}$, we let $m=$ $e^{-\frac{a \xi}{\alpha} \bar{T}}$. From (2.3) and (2.5), we have 


$$
\begin{aligned}
& \left(\frac{h(u)}{u}\right)^{\alpha}=\left(\frac{h(u)}{\bar{h}(u)} \cdot \frac{\bar{h}(u)}{u}\right)^{\alpha} \\
& \left.=\left(\frac{A-h(u)}{m(A-\bar{h}(u))} \cdot \frac{\left(\left(u-\frac{A}{2}\right)^{2}+b^{2}\right)^{\frac{\beta}{2 \alpha}} \exp \left(\frac{\gamma}{b \alpha} \tan ^{-1}\left(\frac{u-\frac{A}{2}}{b}\right)\right)}{\left(\left(\bar{h}(u)-\frac{A}{2}\right)^{2}+b^{2}\right)^{\frac{\beta}{2 \alpha}} \exp \left(\frac{\gamma}{b \alpha} \tan ^{-1}\left(\frac{\bar{h}(u)-\frac{A}{2}}{b}\right)\right)} \cdot e^{-\frac{a \xi}{\alpha} \bar{T}}\right)^{\alpha}\right) \\
& =\left(\frac{A-h(u)}{A-\bar{h}(u)}\right)^{\alpha} \cdot \frac{\left(\left(u-\frac{A}{2}\right)^{2}+b^{2}\right)^{\frac{\beta}{2}} \exp \left(\frac{\gamma}{b} \tan ^{-1}\left(\frac{u-\frac{A}{2}}{b}\right)\right)}{\left(\left(\bar{h}(u)-\frac{A}{2}\right)^{2}+b^{2}\right)^{\frac{\beta}{2}} \exp \left(\frac{\gamma}{b} \tan ^{-1}\left(\frac{\bar{h}(u)-\frac{A}{2}}{b}\right)\right)} .
\end{aligned}
$$

Set

$Y(u)=\left(\left(u-\frac{A}{2}\right)^{2}+b^{2}\right)^{\frac{\beta}{2}} \exp \left(\frac{\gamma}{b} \tan ^{-1}\left(\frac{u-\frac{A}{2}}{b}\right)\right)$,

and

$H(u)=(A-u)^{\alpha} Y(u)$.

Then, we get

$$
\begin{aligned}
\left(\frac{h(u)}{u}\right)^{\alpha} & =\left(\frac{A-h(u)}{A-\bar{h}(u)}\right)^{\alpha} \cdot \frac{Y(u)}{Y(\bar{h}(u))} \\
& =\left(\frac{A-h(u)}{A-u}\right)^{\alpha} \cdot\left(\frac{A-u}{A-\bar{h}(u)}\right)^{\alpha} \cdot \frac{Y(u)}{Y(\bar{h}(u))} \\
& =\left(\frac{A-h(u)}{A-u}\right)^{\alpha} \cdot \frac{H(u)}{H(\bar{h}(u))} .
\end{aligned}
$$

To prove (3.2), it suffices to prove

$$
H(u)<H(\bar{h}(u)), \text { for } u \in(0, A) .
$$

By taking the derivative of $H(u)$, we have

$$
\begin{aligned}
H^{\prime}(u) & =\left(-\frac{\alpha}{A-u}+\frac{\beta\left(u-\frac{A}{2}\right)+\gamma}{\left(u-\frac{A}{2}\right)^{2}+b^{2}}\right) H(u) \\
& =\left(-\frac{\alpha}{A-u}-\frac{\alpha}{u}+\frac{u+c}{u\left(\left(u-\frac{A}{2}\right)^{2}+b^{2}\right)}\right) H(u) \\
& =\frac{1}{u}\left(-\frac{\alpha A}{A-u}+\frac{u+c}{\left(u-\frac{A}{2}\right)^{2}+b^{2}}\right) H(u) \\
& =-\frac{(1+\alpha A)(u-\bar{u})}{(A-u)\left(\left(u-\frac{A}{2}\right)^{2}+b^{2}\right)} H(u),
\end{aligned}
$$

where

$\bar{u}=\frac{c^{*}-c}{1+\alpha A} \leq 0$.

Thus, we have $H^{\prime}(u)<0$, for $u \in(0, A)$. This proves that (3.4) holds since $\bar{h}(u)<u$. Hence, (3.2) is true for the case when $T=T^{*}$.

For the case when $T<T^{*}$, we get $h^{\prime}(0)=$ $e^{(a-\mu)\left(T-T^{*}\right)}<1$ from (2.9), which implies that there is $\delta_{0} \in(0, A)$ such that

$h(u)<u$, for $u \in\left(0, \delta_{0}\right)$.

Now, we show that (3.2) is still true. To this end, we assume by contradiction that (3.2) is not true. Then, there exists $\hat{u} \in\left[\delta_{0}, A\right)$ such that

$h(\hat{u})=\hat{u} \quad$ and $\quad h^{\prime}(\hat{u}) \geq 1$

Let

$$
\begin{aligned}
P(u)= & u^{\alpha}\left(\left(u-\frac{A}{2}\right)^{2}+b^{2}\right)^{\frac{\beta}{2}} \\
& \exp \left(\frac{\gamma}{b} \tan ^{-1}\left(\frac{u-\frac{A}{2}}{b}\right)\right) .
\end{aligned}
$$

Then, we obtain

$$
\begin{aligned}
P^{\prime}(u) & =\left(\frac{\alpha}{u}+\frac{\beta\left(u-\frac{A}{2}\right)+\gamma}{\left(u-\frac{A}{2}\right)^{2}+b^{2}}\right) P(u) \\
& =\frac{u+c}{u\left(\left(u-\frac{A}{2}\right)^{2}+b^{2}\right)} P(u) .
\end{aligned}
$$


From (2.3), we have

$P(\bar{h}(u))=P(u) e^{-a \xi \bar{T}}$,

which, by taking the derivative, yields

$P^{\prime}(\bar{h}(u)) \bar{h}^{\prime}(u)=P^{\prime}(u) e^{-a \xi \bar{T}}$,

which gives, by combining (3.8) with (3.9),

$\bar{h}^{\prime}(u)=\frac{\bar{h}(u)\left(\left(\bar{h}(u)-\frac{A}{2}\right)^{2}+b^{2}\right)(u+c)}{u\left(\left(u-\frac{A}{2}\right)^{2}+b^{2}\right)(\bar{h}(u)+c)}$.

Taking the derivative of both sides of (2.5) yields

$\frac{\bar{h}^{\prime}(u)}{(A-\bar{h}(u))^{2}}=\frac{m h^{\prime}(u)}{(A-h(u))^{2}}$.

Substituting (2.5) and (3.10) into (3.11), we have

$h^{\prime}(u)=\frac{h(u)(A-h(u))\left(\left(\bar{h}(u)-\frac{A}{2}\right)^{2}+b^{2}\right)(u+c)}{u(A-\bar{h}(u))\left(\left(u-\frac{A}{2}\right)^{2}+b^{2}\right)(\bar{h}(u)+c)}$.

From (2.5) again, we get

$$
\begin{aligned}
A-\bar{h}(u) & =\frac{A(A-h(u))}{A-(1-m) h(u)}, \\
\bar{h}(u)+c & =\frac{c A+(m A-(1-m) c) h(u)}{A-(1-m) h(u)}, \\
\bar{h}(u)-\frac{A}{2} & =-\frac{A}{2} \cdot \frac{A-(1+m) h(u)}{A-(1-m) h(u)},
\end{aligned}
$$

which lead to, by (3.12),

where

$$
\begin{aligned}
& B=\frac{a-m \mu}{a \xi}, C=\frac{(1-m) c \mu \xi-2(a-\mu)}{a \xi^{2}}, \text { and } \\
& D=-\frac{\mu\left(c-c^{*}\right)}{a \xi} A .
\end{aligned}
$$

Let the quadratic polynomial $Q(u)$ be defined as:

$Q(u)=B u^{2}+C u+D$.

Then, $Q(\hat{u}) \geq 0$ and $Q(u)$ is concave up since $B>0$. Moreover, we find that $Q(A)=-\frac{a-\mu+a c \xi}{a^{2} \xi^{2}} m \mu A<0$. To sum up, we have

$Q(0)=D \leq 0, Q(\hat{u}) \geq 0$, and $Q(A)<0$,

which is impossible since $0<\hat{u}<A$. Hence, (3.2) is also true for the case when $T<T^{*}$, and thus (3.1) is true. The proof is complete.

The following result shows that if the release amount $c \geq c^{*}$, then the mosquito population will be eradicated eventually, which is guaranteed by the global asymptotical stability of $w_{0}$.

Theorem 3.2 Assume that $c \geq c^{*}$ and $T \leq T^{*}$. Then, the origin $w_{0}$ is globally asymptotically stable.

Proof We first prove that $w_{0}$ is stable. It follows from (2.7) that we can select $\epsilon_{0} \in\left(0, \frac{A}{2}\right)$ such that $\bar{h}(u)>$ $M u$ for $u \in\left(0, \epsilon_{0}\right)$, where $M=\frac{1}{2} e^{-\mu \bar{T}}$. For any $0<$ $\epsilon<\epsilon_{0}$ and $t_{0} \geq 0$, let $\delta=\min \left\{M \epsilon, \frac{m A \epsilon}{A-(1-m) \epsilon}\right\}$, we only need to prove that $u_{0} \in(0, \delta)$ implies

$w\left(t ; t_{0}, u_{0}\right)<\epsilon$, for $t \geq t_{0}$.

It follows from (3.7) that

$$
h^{\prime}(\hat{u})=\frac{\left[\frac{A^{2}}{4}(A-(1+m) \hat{u})^{2}+b^{2}(A-(1-m) \hat{u})^{2}\right](\hat{u}+c)}{A\left(\left(\hat{u}-\frac{A}{2}\right)^{2}+b^{2}\right)[c A+(m A-(1-m) c) \hat{u}]}
$$$$
\geq 1 \text {, }
$$

which yields

$$
B \hat{u}^{2}+C \hat{u}+D \geq 0,
$$

For convenience, we denote $w(t)=w\left(t ; t_{0}, u_{0}\right)$. Since model (1.4)-(1.5) is $T$-periodic, we may assume $t_{0} \in[0, T)$.

We now prove (3.16) by contradiction. If (3.16) does not hold, then there is some $\bar{t}>t_{0}$ such that

$w(\bar{t})=\epsilon, \quad w(t)<\epsilon, \quad$ for $t \in\left[t_{0}, \bar{t}\right)$. 
Let $p$ be a positive integer such that $\bar{t} \in[p T,(p+1) T)$. Then, there are three possible cases to consider: (I) $\bar{t}=p T$, (II) $\bar{t}=p T+\bar{T}$, and (III) $\bar{t} \in(p T, p T+\bar{T}) \cup$ $(p T+\bar{T},(p+1) T)$. Obviously, (II) is impossible since $w(p T+\bar{T})$ is the minimum of $w(t)$ for $t \in[p T,(p+$ 1)T). Now, we show that (I) is impossible. In fact, if (I) holds, then either $p=1$ or $p>1$.

For the case when $p=1$, there are two possible cases $t_{0} \in[0, \bar{T}]$ and $t_{0} \in(\bar{T}, T)$ to consider.

Assume $t_{0} \in[0, \bar{T}]$. Then, we have from (2.5),

$w(\bar{T})=\frac{A m w(T)}{A-(1-m) w(T)}=\frac{A m \epsilon}{A-(1-m) \epsilon} \geq \delta$,

which yields $w\left(t_{0}\right) \geq w(\bar{T}) \geq \delta$, a contradiction to $w\left(t_{0}\right)=u_{0} \in(0, \delta)$.

Assume $t_{0} \in(\bar{T}, T)$. Then, we have, by integrating (2.4) from $t_{0}$ to $\bar{t}$,

$\frac{w\left(t_{0}\right)}{A-w\left(t_{0}\right)}=\frac{\bar{m} w(\bar{t})}{A-w(\bar{t})}$,

which leads to

$w\left(t_{0}\right)=\frac{A \bar{m} w(\bar{t})}{A-(1-\bar{m}) w(\bar{t})}=\frac{A \bar{m} \epsilon}{A-(1-\bar{m}) \epsilon}$,

where $\bar{m}=e^{-a A \xi\left(\bar{t}-t_{0}\right)}>m$. Since $\frac{A \epsilon x}{A-(1-x) \epsilon}$ is strictly increasing with respect to $x \in(0,1)$, we obtain

$w\left(t_{0}\right)>\frac{A m \epsilon}{A-(1-m) \epsilon} \geq \delta$,

a contradiction again. This shows that (I) is impossible for the case when $p=1$.

For the case when $p>1$, we see that $w(p T)=$ $\epsilon>w((p-1) T)$ by the fact that $t_{0}<T \leq(p-1) T$. Let $u_{1}=w((p-1) T)$. Then $h\left(u_{1}\right)=w\left(T ; 0, u_{1}\right)=$ $w(p T)>u_{1}$. From (3.1), we have $h\left(u_{1}\right)<u_{1}$, a contradiction. This shows that (I) is impossible for the case when $p>1$. Hence, (I) is impossible.

We next show that (III) is impossible as well. By (3.17), we have $w^{\prime}(\bar{t}) \geq 0$, which excludes the situation of $\bar{t} \in(p T, p T+\bar{T})$. Thus, we only need to consider the case of $\bar{t} \in(p T+\bar{T},(p+1) T)$. From equation (1.5), we see that $w(t)$ is strictly increasing for $t \in$ $(p T+\bar{T},(p+1) T)$, and hence $w((p+1) T)>w(\bar{t})=$ $\epsilon>w(p T)$. From Lemma 2.1, we get $h(u)>u$, which contradicts (3.1). Hence, (III) is also impossible and so (3.16) is true. This shows that $w_{0}$ is stable.

Finally, we prove that $w_{0}$ is globally attractive. For any $t_{0} \in[0, T)$ and $u_{0}>0$, we just need to show

$\lim _{t \rightarrow \infty} w(t)=\lim _{t \rightarrow \infty} w\left(t ; t_{0}, u_{0}\right)=0$.
Since $w(t)$ reaches its maximum at $n T$ for $t \in[(n-$ 1) $T+\bar{T}, n T+\bar{T}], n=2,3, \ldots$, it suffices to prove that

$$
\begin{aligned}
\lim _{n \rightarrow \infty} h_{n}(u) & =\lim _{n \rightarrow \infty} w(n T) \\
& =\lim _{n \rightarrow \infty} w\left(n T ; t_{0}, u_{0}\right)=0 .
\end{aligned}
$$

From (3.1) and Lemma 2.1, we see that sequence $\left\{h_{n}(u)\right\}$ is strictly decreasing, so $\operatorname{limit}_{\lim } \rightarrow \infty h_{n}(u)=$ $q \geq 0$ exists. It is easy to prove $q=0$ due to the non-existence of periodic solution to model(1.4)-(1.5). Hence, (3.18) is true and the proof is complete.

We then give a numerical example to illustrate our theoretical results in Theorem 3.2.

Example 1 Let the parameters $a, \mu, \xi$ and $\bar{T}$ be given as follows:

$a=2, \quad \mu=0.05, \quad \xi=0.001, \quad$ and $\quad \bar{T}=14$.

Then, $g^{*}=9506.25, c^{*}=39,000$, and $T^{*} \approx 14.36$. By taking $c=40,000>c^{*}$ and $T=14.10<T^{*}$, $c=c^{*}$ and $T=14.1<T^{*}, c=40,000>c^{*}$ and $T=T^{*}$, and $c=c^{*}$ and $T=T^{*}$, respectively, the conditions of Theorem 3.2 are all satisfied and $w_{0}$ is globally asymptotically stable as shown in panels (A), (B), (C) and (D) of Fig. 1.

Theorem 3.2 tells us that the release strategy of $c \geq$ $c^{*}$ and $T \leq T^{*}$ can eventually ensure the elimination of mosquitoes. The next theorem focuses on the situation when $c \in\left(g^{*}, c^{*}\right)$.

Theorem 3.3 Assume that $g^{*}<c<c^{*}$. Then, the following two conclusions hold.

(1) If $T=T^{*}$, then model (1.4)-(1.5) has a unique $T$ periodic solution, which is globally asymptotically stable.

(2) The origin is asymptotically stable if and only if $T<T^{*}$.

Proof (1) Assume that $T=T^{*}$. We need to show that model (1.4)-(1.5) has a unique $T$-periodic solution, which is globally asymptotically stable. From (3.6), we have $0<\bar{u}=\frac{c^{*}-c}{1+\alpha A}<A$ since $c<c^{*}$. We first claim that

$h(u)>u, \quad$ for $u \in(0, \bar{u})$. 

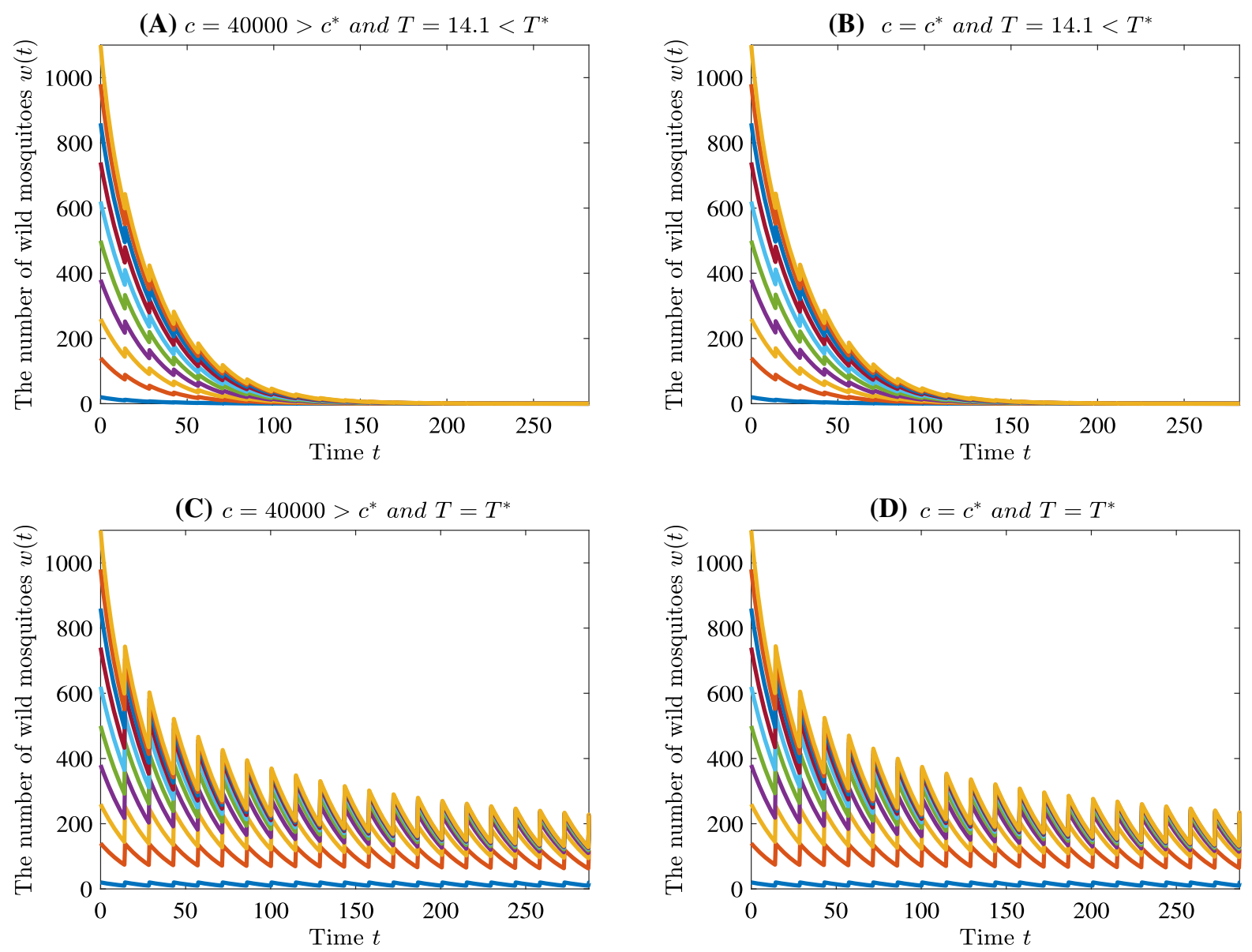

Fig. 1 For given parameters in (3.19), the above four figures, which correspond to four kinds of sub-cases of the conditions $c \geq c^{*}$ and $T \leq T^{*}$, are used to support our theoretical results in Theorem 3.2

From (3.3), we just need to show

$$
H(u)>H(\bar{h}(u)), \text { for } u \in(0, \bar{u}) \text {. }
$$

From (3.5), we reach

$$
\begin{aligned}
& H^{\prime}(u)>0, \quad \text { for } 0<u<\bar{u}, \quad \text { and } \\
& H^{\prime}(u)<0, \text { for } u \in(\bar{u}, A),
\end{aligned}
$$

which means that $H(u)$ is strictly increasing for $u \in$ $(0, \bar{u})$, and strictly decreasing for $u \in(\bar{u}, A)$. Then, (3.21) holds as $\bar{h}(u)<u<\bar{u}$. Hence, (3.20) is true. Combining (2.6) with (3.20), we know that there must exist $u_{1} \in(\bar{u}, A)$ such that

$h\left(u_{1}\right)=u_{1}$, and $h(u)>u$, for $u \in\left(0, u_{1}\right)$.

Thus, $w\left(t ; 0, u_{1}\right)$ is a $T$-periodic solution of model (1.4)-(1.5). This shows the existence of a $T$-periodic solution.
Now, we prove the uniqueness of $T$-periodic solutions of model (1.4)-(1.5) by contradiction. Assume that model (1.4)-(1.5) has another $T$-periodic solution. Then, there is some $u_{2} \in\left(u_{1}, A\right)$ such that $h\left(u_{2}\right)=u_{2}$ and $h(u)<u$ for $u \in\left(u_{2}, A\right)$. From (3.3), we have

$H\left(u_{i}\right)=H\left(\bar{h}\left(u_{i}\right)\right), \quad i=1,2$,

which gives, along with (3.22) and the fact of $\bar{u}<u_{1}<$ $u_{2}<A$,

$\bar{h}\left(u_{2}\right)<\bar{h}\left(u_{1}\right)<\bar{u}$.

Note that $\bar{h}(u)$ is strictly increasing, we have $\bar{h}\left(u_{2}\right)>$ $\bar{h}\left(u_{1}\right)$, which leads to a contradiction. Hence, model (1.4)-(1.5) admits $w\left(t ; 0, u_{1}\right)$ as its unique $T$-periodic solution.

To prove the global asymptotical stability of $w(t ; 0$, $u_{1}$ ), we only need to prove $w_{0}$ is unstable. From (3.20) 
and Lemma 2.1, we find that both $\left\{h_{n}(u)\right\}$ and $\left\{\bar{h}_{n}(u)\right\}$ are strictly increasing for $u \in(0, \bar{u})$, which shows that $w_{0}$ is unstable. Hence, the proof of (1) is complete.

(2) Assume that $w_{0}$ is asymptotically stable. We need to show $T<T^{*}$. From (2.9), we have $h^{\prime}(0)=$ $e^{(a-\mu)\left(T-T^{*}\right)}$. It suffices to verify $h^{\prime}(0)<1$. Assume by contradiction that $h^{\prime}(0) \geq 1$. For case $h^{\prime}(0)>1$, there exists $\delta^{*}>0$ small enough such that $h(u)>u$ for $u \in\left(0, \delta^{*}\right)$, which implies that, from Lemma 2.1, $\left\{h_{n}(u)\right\}$ is strictly increasing for $u \in\left(0, \delta^{*}\right)$, and thus $\lim _{n \rightarrow \infty} h_{n}(u) \neq 0$, a contradiction to the asymptotical stability of $w_{0}$. Similarly, for case $h^{\prime}(0)=1$, we obtain $T=T^{*}$, which yields a unique globally asymptotically stable $T$-periodic solution from the first conclusion of this theorem. Again, a contradiction to the asymptotical stability of $w_{0}$.

Next, we assume $T<T^{*}$. We need to prove that $w_{0}$ is asymptotically stable. From (2.9), we see $h^{\prime}(0)=$ $e^{(a-\mu)\left(T-T^{*}\right)}<1$, which means that there exists $\delta_{1}>$ 0 small enough, such that

$h(u)<u$, for $u \in\left(0, \delta_{1}\right)$.

For any $\epsilon \in\left(0, \frac{\delta_{1}}{2}\right)$, let $\delta=\min \left\{\epsilon, \frac{m A \epsilon}{A-(1-m) \epsilon}\right\}$. Then, by a similar argument to that of Theorem 3.2, we can prove that $w_{0}$ is asymptotically stable. The proof is finished.

For the critical release period $T=T^{*}$, Theorem 3.3(1) shows that if $g^{*}<c<c^{*}$, then mosquito population eradication is impossible due to the existence of a unique globally asymptotically stable $T$-periodic solution. To see this, we give a numerical example in the following to illustrate our theoretical results in Theorem 3.3, together with an observation on the existence of exactly two periodic solutions for some $T<T^{*}$ and $c \in\left(g^{*}, c^{*}\right)$ with the theoretical proofs pending.

Example 2 For given parameters in (3.19), we have

$g^{*}=9506.25, \quad c^{*}=39,000, \quad$ and $T^{*} \approx 14.36$.

By taking $c=10,000 \in\left(g^{*}, c^{*}\right)$ and $T=T^{*}$, panel (A) of Fig. 2 shows that no matter how small the initial population size is, solutions of model (1.4)-(1.5) are attracted by the unique $T$-periodic solution.

The situation for the case when $c \in\left(g^{*}, c^{*}\right)$ and $T<T^{*}$, however, is quite tricky. We can only prove the asymptotical stability of $w_{0}$ theoretically, but fail to count the exact number of $T$-periodic solutions of model (1.4)-(1.5). To numerically explore this problem, let $c=10,000 \in\left(g^{*}, c^{*}\right)$ and $T=14.10<$ $T^{*}$. Then, $w_{0}$ is asymptotically stable from Theorem 3.3(2). Moreover, there exists a asymptotically stable $T$-periodic solution as shown in panel (B) of Fig. 2. Thus, we deduce that model (1.4)-(1.5) has exactly two $T$-periodic solutions, the larger one being asymptotically stable, and the smaller one unstable, which has not been proved theoretically at the present time.

From Theorem 3.3, it is easy to see that the following corollary is true.

Corollary 3.4 Assume that $g^{*}<c<c^{*}$ and $T \leq$ $T^{*}$. Then, model (1.4)-(1.5) has a unique T-periodic solution, which is globally asymptotically stable, if and only if $T=T^{*}$.

\subsection{Release less often with $T>T^{*}$}

In this subsection, we consider the case of $T>T^{*}$, which means that we release sterile mosquitoes less often. For this case, we have the following theorem.

Theorem 3.5 Assume that $c>g^{*}$ and $T>T^{*}$. Then, model (1.4)-(1.5) has a unique T-periodic solution, which is globally asymptotically stable.

Proof From (2.9), we reach $h^{\prime}(0)>1$, which indicates that $h(u)>u$ for $u \in(0, \delta)$, where $\delta$ is sufficiently small. This, together with (2.6), means that there exists $u_{1} \in[\delta, A)$ such that

$$
\begin{aligned}
& h\left(u_{1}\right)=u_{1}, h^{\prime}\left(u_{1}\right) \leq 1, \quad \text { and } \\
& h(u)>u, \text { for } u \in\left(0, u_{1}\right) .
\end{aligned}
$$

Assume by contradiction that model (1.4)-(1.5) has another $T$-periodic solution. Then, there is $u_{2} \in$ $\left(u_{1}, A\right)$ such that

$$
\begin{aligned}
& h\left(u_{2}\right)=u_{2}, h^{\prime}\left(u_{2}\right) \leq 1, \text { and } \\
& h(u)<u, \text { for } u \in\left(u_{2}, A\right) .
\end{aligned}
$$

Hence, we have

$h\left(u_{i}\right)=u_{i}, \quad$ and $\quad h^{\prime}\left(u_{i}\right) \leq 1, i=1,2$,

which consists of the following four cases:

$$
\begin{aligned}
& h^{\prime}\left(u_{1}\right)=1, \quad \text { and } \quad h^{\prime}\left(u_{2}\right)=1, \\
& h^{\prime}\left(u_{1}\right)<1, \quad \text { and } \quad h^{\prime}\left(u_{2}\right)=1, \\
& h^{\prime}\left(u_{1}\right)<1, \quad \text { and } \quad h^{\prime}\left(u_{2}\right)<1,
\end{aligned}
$$



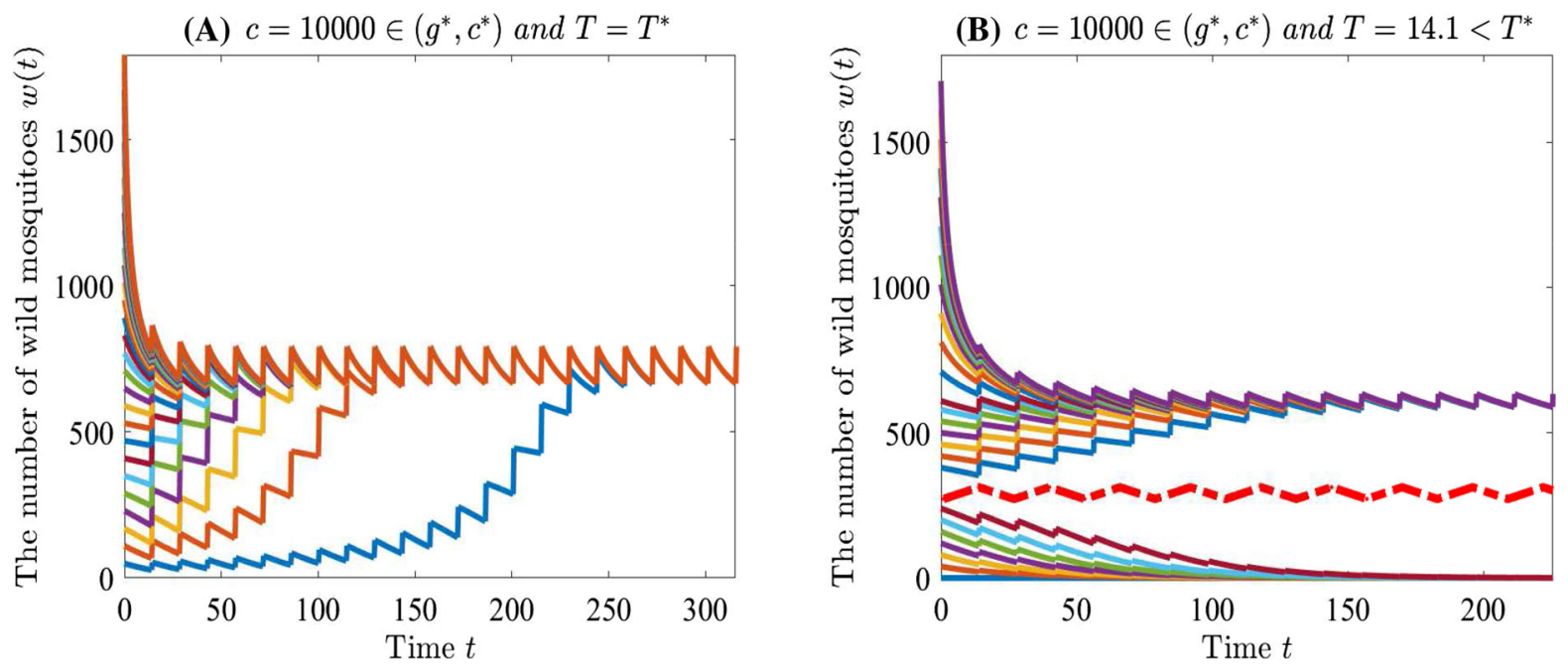

Fig. 2 For given parameters in (3.19), we obtain the above numerical illustration, where panel $\mathbf{A}$ and panel $\mathbf{B}$ are for Theorem 3.3(1) and Theorem 3.3(2), respectively, and the red dashed fold line in panel $\mathbf{B}$ represents the unstable $T$-periodic solution

and

$h^{\prime}\left(u_{1}\right)=1$, and $h^{\prime}\left(u_{2}\right)<1$.

From (3.13) and (3.23), the above four cases are equivalent to

$Q\left(u_{1}\right)=0$, and $Q\left(u_{2}\right)=0$,

$Q\left(u_{1}\right)<0$, and $Q\left(u_{2}\right)=0$,

$Q\left(u_{1}\right)<0$, and $Q\left(u_{2}\right)<0$,

and

$Q\left(u_{1}\right)=0$, and $Q\left(u_{2}\right)<0$,

respectively, where $B, C, D$ are defined in (3.14), and $Q(u)$ is defined in (3.15).

Since $Q(A)<0$ and $B=\frac{a-m \mu}{a \xi}>0$, we can easily see that (3.24) and (3.25) are impossible.

Now, we show that (3.26) is impossible. In fact, if (3.26) holds, then by (3.13) and (3.23), there is $\tilde{u} \in$ $\left(u_{1}, u_{2}\right)$ such that

$h(\widetilde{u})=\widetilde{u}, \quad$ and $\quad h^{\prime}(\widetilde{u}) \geq 1$,

which leads to $Q(\widetilde{u}) \geq 0$. However, from $B>0$ and $\tilde{u} \in\left(u_{1}, u_{2}\right)$, along with (3.26), we must have $Q(\tilde{u})<$ 0 , a contradiction. Thus, (3.26) is impossible as well.

Finally, we verify that (3.27) is also impossible. To this end, let $k-1>0$ be small enough such that $h(u)-k u=0$ has three roots, denoted by $v_{i}(k)=$ $v_{i}, i=1,2,3$, with

$0<v_{1}<u_{1}<v_{2}<v_{3}<u_{2}<A$,

and

$\begin{array}{ll}h\left(v_{1}\right)=k v_{1}, & h^{\prime}\left(v_{1}\right) \leq k, \\ h\left(v_{2}\right)=k v_{2}, & h^{\prime}\left(v_{2}\right) \geq k, \\ h\left(v_{3}\right)=k v_{3}, & h^{\prime}\left(v_{3}\right) \leq k .\end{array}$

Substituting (3.28) in (3.13), we obtain

$h^{\prime}\left(v_{1}\right)$

$$
=\frac{k\left[\frac{A^{2}}{4}\left(A-(1+m) k v_{1}\right)^{2}+b^{2}\left(A-(1-m) k v_{1}\right)^{2}\right]\left(v_{1}+c\right)}{A\left(\left(v_{1}-\frac{A}{2}\right)^{2}+b^{2}\right)\left[c A+(m A-(1-m) c) k v_{1}\right]}
$$

$\leq k$,

$h^{\prime}\left(v_{2}\right)$

$=\frac{k\left[\frac{A^{2}}{4}\left(A-(1+m) k v_{2}\right)^{2}+b^{2}\left(A-(1-m) k v_{2}\right)^{2}\right]\left(v_{2}+c\right)}{A\left(\left(v_{2}-\frac{A}{2}\right)^{2}+b^{2}\right)\left[c A+(m A-(1-m) c) k v_{2}\right]}$

$\geq k$

$h^{\prime}\left(v_{3}\right)$

$=\frac{k\left[\frac{A^{2}}{4}\left(A-(1+m) k v_{3}\right)^{2}+b^{2}\left(A-(1-m) k v_{3}\right)^{2}\right]\left(v_{3}+c\right)}{A\left(\left(v_{3}-\frac{A}{2}\right)^{2}+b^{2}\right)\left[c A+(m A-(1-m) c) k v_{3}\right]}$ $\leq k$,

which are equivalent to

$$
\begin{aligned}
& B(k) v_{1}^{2}+C(k) v_{1}+D(k) \leq 0, \\
& B(k) v_{2}^{2}+C(k) v_{2}+D(k) \geq 0, \\
& B(k) v_{3}^{2}+C(k) v_{3}+D(k) \leq 0,
\end{aligned}
$$


respectively, where

$$
\begin{aligned}
B(k)= & k\left(\frac{k(1-m)^{2}}{\alpha} c+(k-1) m A^{2}+(1-m) c A\right), \\
C(k)= & \frac{k^{2}(1-m)^{2}}{\alpha} c^{2}+(k+1)(m k-1) c A^{2} \\
& +\frac{2 k(m-1)}{\alpha} c A, \\
D(k)= & -\frac{k(1-m)}{\alpha} c A\left(c-\frac{1-m k}{k-m k} c^{*}\right) .
\end{aligned}
$$

Let

$Q_{k}(u)=B(k) u^{2}+C(k) u+D(k)$.

Then, (3.29) becomes

$Q_{k}\left(v_{1}\right) \leq 0, Q_{k}\left(v_{2}\right) \geq 0$, and $Q_{k}\left(v_{3}\right) \leq 0$,

which leads to a contradiction, since $B(k)>0$ and $Q_{k}(u)$ is a quadratic function. Hence, (3.27) is also impossible. This shows that $T$-periodic solution of model (1.4)-(1.5) is unique.

By a similar arguments used in the proof of Theorem 3.3(1), we can prove the global asymptotical stability of the unique $T$-periodic solution. The proof is complete.

Combining Theorems 3.2 with 3.5, we obtain the following corollary.

Corollary 3.6 Assume that $c \geq c^{*}$. Then, the origin is globally asymptotically stable if and only if $T \leq T^{*}$.

\section{Concluding remarks}

Combining the modeling ideas for the interactive wild and sterile mosquitoes in [17], in which only the sexually active sterile mosquitoes are considered, in [29], in which the density dependent survival probability is assumed, and in $[19,25,26]$, in which the releases of the sterile mosquitoes are impulsive, constant and periodic, we formulate and investigate model (1.4)-(1.5) in this paper. In [29], the author supposed that the release function $g$ of sterile mosquitoes is an independent variable satisfying an independent dynamical equation, see also [1,32]. In [30], the authors treated the release function $g$ as a given nonnegative continuous function in advance, especially, a constant function related to the release strategy of $T=\bar{T}$.

Although model (1.4)-(1.5) looks simple, it has rich and complex dynamics including the local and global stability of the origin, the existence, stability and semistability of a unique $T$-periodic solution, and the existence and stability of exact two $T$-periodic solutions. We have found the corresponding threshold values such as $T^{*}$ for the waiting period between two consecutive releases, $g^{*}$ and $c^{*}$ for the release amount of the sterile mosquitoes each time. We have shown that the origin is asymptotically stable provided $c \geq c^{*}$ and $T \leq T^{*}$, or $c \in\left(g^{*}, c^{*}\right)$ and $T<T^{*}$ in Theorem 3.2 and Theorem 3.3(2), respectively, and model (1.4)-(1.5) admits a unique globally asymptotically stable $T$-periodic solution, provided $c \in\left(g^{*}, c^{*}\right)$ and $T=T^{*}$ or $c>g^{*}$ and $T>T^{*}$ in Theorem 3.3(1) and Theorem 3.5, respectively. Figure 1 verifies all conditions in Theorem 3.2 by four cases: (i) $c>c^{*}$ and $T<T^{*}$, (ii) $c=c^{*}$ and $T<T^{*}$, (iii) $c>c^{*}$ and $T=T^{*}$, and (iv) $c=c^{*}$ and $T=T^{*}$. Figure 2A, B verifies Theorem 3.3(1) and Theorem 3.3(2), respectively. We should mention here that for the case $c \in\left(g^{*}, c^{*}\right)$ and $T<T^{*}$, it is possible for model (1.4)-(1.5) to have exact two $T$-periodic solutions, or a unique semi-stable $T$-periodic solution, or the origin is GAS. We leave this situation as our future work. Furthermore, it remains unknown for the case of $c \in\left(0, g^{*}\right]$, which will be what we are going to study in the future, and by numerical simulations, we see that model (1.4)-(1.5) may have two $T$-periodic solutions, with the smaller one unstable and the bigger one stable.

Author contributions All authors contributed to the conception and design of the study. Material preparation was performed by Yantao Shi and Rong Yan, and analysis was performed by Zhongcai Zhu, Bo Zheng and Jianshe Yu. All authors read and approved the submission.

Funding This work is supported by National Natural Science Foundation of China (12071095, 11971127, 11631005), Program for Changjiang Scholars and Innovative Research Team in University (IRT_16R16) and Innovative Research Grant for the Postgraduates of Guangzhou University (2019GDJC-D05).

\section{Declaration}

Conflict of interest The authors declare that they have no conflict of interest.

Open Access This article is licensed under a Creative Commons Attribution 4.0 International License, which permits use, sharing, adaptation, distribution and reproduction in any medium or format, as long as you give appropriate credit to the original author(s) and the source, provide a link to the Creative Commons licence, and indicate if changes were made. The images or 
other third party material in this article are included in the article's Creative Commons licence, unless indicated otherwise in a credit line to the material. If material is not included in the article's Creative Commons licence and your intended use is not permitted by statutory regulation or exceeds the permitted use, you will need to obtain permission directly from the copyright holder. To view a copy of this licence, visit http://creativecommons.org/licenses/ by/4.0/.

\section{References}

1. Cai, L., Ai, S., Li, J.: Dynamics of mosquitoes populations with different strategies for releasing sterile mosquitoes. SIAM J. Appl. Math. 74, 1786-1809 (2014)

2. Hu, L., Huang, M., Tang, M., Yu, J., Zheng, B.: Wolbachia spread dynamics in stochastic environments. Theor. Popul. Biol. 106, 32-44 (2015)

3. Huang, M., Luo, J., Hu, L., Zheng, B., Yu, J.: Assessing the efficiency of Wolbachia driven Aedes mosquito suppression by delay differential equations. J. Theor. Biol. 440, 1-11 (2018)

4. Liu, Y., Jiao, F., Hu, L.: Modeling mosquito population control by a coupled system. J. Math. Anal. Appl. 506, 125671 (2022)

5. Li, J.: Differential equations models for interacting wild and transgenes and Allee effects. J. Biol. Dyn. 2, 241-258 (2008)

6. Zheng, B., Tang, M., Yu, J., Qiu, J.: Wolbachia spreading dynamics in mosquitoes with imperfect maternal transmission. J. Math. Biol. 76, 235-263 (2018)

7. Hu, L., Tang, M., Wu, Z., Xi, Z., Yu, J.: The threshold infection level for Wolbachia invasion in random environments. J. Differ. Equ. 266(7), 4377-4393 (2019)

8. Huang, M., Tang, M. Yu, J.: Wolbachia infection dynamics by reaction-diffusion equations. Sci. China Math. 58(1), 77$96(2015)$

9. Li, J.: Simple mathematical models for interacting wild and transgenic mosquito populations. Math. Biosci. 189, 39-59 (2004)

10. Ai, S., Li, J., Yu, J., Zheng, B.: Stage-structured models for interactive wild and periodically and impulsively released sterile mosquitoes. Discrete Contin. Dyn. Syst. Ser. B. https://doi.org/10.3934/dcdsb.2021172

11. Li, J., Cai, L., Li, Y.: Stage-structured wild and sterile mosquito population models and their dynamics. J. Biol. Dyn. 11, 79-101 (2017)

12. Li, Y., Liu, X.: An impulsive model for Wolbachia infection control of mosquito-borne diseases with general birth and death rate functions. Nonlinear Anal. Real World Appl. 37, 412-432 (2017)

13. Li, Y., Liu, X.: A sex-structured model with birth pulse and release strategy for the spread of Wolbachia in mosquito population. J. Theor. Biol. 448, 53-65 (2018)

14. Li, Y., Liu, X.: Modeling and control of mosquito-borne diseases with Wolbachia and insecticides. Theor. Popul. Biol. 132, 82-91 (2020)

15. Shi, Y., Yu, J.: Wolbachia infection enhancing and decaying domains in mosquito population based on discrete models. J. Biol. Dyn. 14(1), 679-695 (2020)
16. Shi, Y., Zheng, B.: Discrete dynamical models on Wolbachia infection frequency in mosquito populations with biased release ratios. J. Biol. Dyn. (2021). https://doi.org/10.1080/ 17513758.2021.1977400

17. Yu, J.: Modeling mosquito population suppression based on delay differential equations. SIAM J. Appl. Math. 78, 31683187 (2018)

18. Yu, J., Li, J.: Dynamics of interactive wild and sterile mosquitoes with time delay. J. Biol. Dyn. 13, 606-620 (2019)

19. Yu, J.: Existence and stability of a unique and exact two periodic orbits for an interactive wild and sterile mosquito model. J. Differ. Equ. 269, 10395-10415 (2020)

20. Yu, J., Zheng, B.: Modeling Wolbachia infection in mosquito population via discrete dynamical models. J. Differ. Equ. Appl. 25(11), 1549-1567 (2019)

21. Zheng, B., Yu, J.: Existence and uniqueness of periodic orbits in a discrete model on Wolbachia infection frequency. Adv. Nonlinear Anal. 11, 212-224 (2022)

22. Zheng, B., Li, J., Yu, J.: One discrete dynamical model on Wolbachia infection frequency in mosquito populations. Sci. China Math. (2021). https://doi.org/10.1007/ s11425-021-1891-7

23. Zheng, B., Yu, J., Xi, Z., Tang, M.: The annual abundance of dengue and Zika vector Aedes albopictus and its stubbornness to suppression. Ecol. Model. 387(10), 38-48 (2018)

24. Zhang, X., Liu, Q., Zhu, H.: Modeling and dynamics of Wolbachia-infected male releases and mating competition on mosquito control. J. Math. Biol. 81, 243-276 (2020)

25. Yu, J., Li, J.: Global asymptotic stability in an interactive wild and sterile mosquito model. J. Differ. Equ. 269, 61936215 (2020)

26. Zheng, B., Yu, J., Li, J.: Modeling and analysis of the implementation of the Wolbachia incompatible and sterile insect technique for mosquito population suppression. SIAM J. Appl. Math. 81, 718-740 (2021)

27. Liu, F., Yao, C., Lin, P., Zhou, C.: Studies on life table of the natural population of Aedes albopictus. Acta Sci. Natur. Univ. Sunyatseni 31, 84-93 (1992)

28. CDC, Life cycle: The mosquito. https://www.cdc.gov/ dengue/resources/factsheets/mosquitolifecyclefinal.pdf (2019)

29. Li, J.: New revised simple models for interactive wild and sterile mosquito populations and their dynamics. J. Biol. Dyn. 11, 316-333 (2017)

30. Lin, G., Hui, Y.: Stability analysis in a mosquito population suppression model. J. Biol. Dyn. 14, 578-589 (2020)

31. Li, J., Ai, S.: Impulsive releases of sterile mosquitoes and interactive dynamics with time delay. J. Biol. Dyn. 14, 313331 (2020)

32. Cai, L., Ai, S., Fan, G.: Dynamics of delayed mosquitoes populations models with two different strategies of releasing sterile mosquitoes. Math. Biosci. Eng. 15, 1181-1202 (2018)

33. Zheng, B., Tang, M., Yu, J.: Modeling Wolbachia spread in mosquitoes through delay differential equations. SIAM J. Appl. Math. 74, 743-770 (2014)

34. Sharma, S., Samanta, G.: Stability analysis and optimal control of an epidemic model with vaccination. Int. J. Biomath. 8, 1550030 (2015) 
35. Hirsch, M., Smale, S., Devaney, R.: Differential Equations, Dynamical Systems, and an Introduction to Chaos, 2nd edn. Academic Press, Orlando (2004)

Publisher's Note Springer Nature remains neutral with regard to jurisdictional claims in published maps and institutional affiliations. 